\title{
Evaluation of two rapid methods for identifying and biotyping Haemophilus influenzae
}

\author{
P G Murphy, I Craig, A C Lafong, E T M Smyth
}

\begin{abstract}
Several rapid method kits (one to four hours) have become available for the identification of Haemophilus and related genera. Two kits (the "Rapid NH" system and the "RIM Haemophilus" system), which include the identification and biotyping of $\boldsymbol{H}$ influenzae, were investigated for the rapid identification and biotyping of 193 isolates of $H$ influenzae and the results compared with those obtained by more standard overnight methods. The kits were convenient to use and gave reliable and rapid speciation of all isolates. Both test systems were unreliable for biotyping: 42 isolates were wrongly biotyped by the RIM kit and 40 isolates wrongly biotyped by the rapid NH kit.

It is concluded that the test kits may be useful for the rapid identification of $H$ influenzae but that they are not reliable for the biotyping of this species.
\end{abstract}

Biotyping is a useful epidemiological tool and gives better resolution of types for both invasive and non-invasive strains than Pittman capsular typing. ${ }^{12}$ The traditional biotyping system based on urea hydrolysis, ornithine decarboxylation, and indole production differentiates eight biotypes of $H$ influenzae and four biotypes of $H$ para influenzae. ${ }^{34}$ The preparation of reagents used in this method is laborious and time consuming, and several kits giving same day results have been developed. A modification of the API 10S kit has been shown to give $100 \%$ correlation with conventional biotyping techniques but this is also an overnight method. Traditional speciation of Haemophilus relies on the demonstration of " $x$ " and " $v$ " factor growth dependence which is an overnight test and can sometimes be difficult to interpret if a washing procedure is omitted due to " $x$ " factor carry over from a primary culture on blood agar. We have used " $x$ " and " $v$ " factor growth dependence and the API $10 \mathrm{~S}$ as reference methods to test the performance of two rapid method kits.

\section{Methods} The test organisms were routine clinical Moraxella, and related groups of organisms. isolates provided by the bacteriology depart- This kit consists of a 10 well panel, permitting ments of three hospitals in Northern Ireland 12 biochemical tests to detect phosphate between 1985 and 1986. Colonies were hydrolysis, nitrate reduction, ONPG isolated from chocolate agar plates after over- hydrolysis, prolyl-p-nitroanilide hydrolysis, $\gamma$

night incubation in $5-7^{\circ}$ o carbon dioxide at $37^{\circ} \mathrm{C}$. Single colonies were suspended in $2 \mathrm{ml}$ peptone water to reduce carry over of " $x$ " factor and then subcultured on to nutrient agar to determine growth dependence on " $x$ " and " $v$ " factors. A purity plate was also inoculated from the peptone water inoculum on to chocolate blood agar. National Collection of Type Cultures (NCTC) strains of $H$ influenzae biotypes $\mathrm{I}-\mathrm{V}$ were used as control organisms. The test systems were inoculated from overnight growth from purity plates according to the manufacturer's instructions (a $1 \mu$ l loopful inoculum for the RIM tubes and a McFarland No 3 inoculum for the Rapid NH wells). Pittman capsular serotyping was performed on the $H$ influenzae strains using agglutination with specific antibody (Wellcome) absorbed to latex particles (Difco). ${ }^{6}$ The method used for the modified API 10S system (API-bioMèrieux, UK) was that of Mehtar but simplified. ${ }^{57}$ Only the indole production, urea hydrolysis, and ornithine decarboxylation were used as reference reactions for biotyping. All reactions were complete after overnight incubation at $37^{\circ} \mathrm{C}$

The Haemophilus kits $\mathrm{H} 1$ and $\mathrm{H} 2$ of the RIM series (Austin Biological Labs, Texas, USA) were used for identification and biotyping, respectively. The test reactions are based on microbial degradation of specific substrates detected by various indicator systems. The two kits, $\mathrm{H} 1$ and $\mathrm{H} 2$, are used together and may be supplemented by oxidase and catalase reactions to differentiate Haemophilus and some related organisms. The $\mathrm{H} 1$ kit uses porphyrin synthesis and dextrose, lactose, and sucrose utilisation to speciate Haemophilus. The porphyrin synthesis was detected by a lower phase colour change after the addition of Kovac's reagent, while an upper phase colour change indicated indole production. A second tube was used as a negative control as no Kovac's reagent was added. The $\mathrm{H} 2$ kit tested indole production, urea hydrolysis, and ornithine decarboxylation. All reactions in the RIM kit were complete after two hours' incubation at $37^{\circ} \mathrm{C}$ in air, some as quickly as after 30 minutes' incubation. The other kit tested was the Rapid NH system (Innovative Diagnostic Systems Inc, USA) which is also marketed to speciate Neisseria Kingella, Bacteriology, Royal Belfast

E T M Smyth

Correspondence to: Dr P G Murphy

Accepted for publication 23 January 1990 
Table 1 Biotypes of H influenzae and their distribution

\begin{tabular}{lllll}
\hline Biotype & Indole & Urea & Ornithine & Distribution (\%) \\
\hline I & + & + & + & $67 / 193(35)$ \\
II & + & + & - & $62 / 193(32)$ \\
III & - & + & - & $38 / 193(20)$ \\
IV & - & + & + & $5 / 193(3)$ \\
V & + & - & + & $17 / 193(9)$ \\
VI & - & - & + & $2 / 193(1)$ \\
VII & + & - & - & $1 / 193(0.5)$ \\
VIII & - & - & - & $1 / 193(0.5)$ \\
\hline
\end{tabular}

glutamyl-p-nitroanilide hydrolysis, resazurin reduction, glucose and sucrose utilisation, penicillin hydrolysis by $\beta$ lactamase, indole production, urea hydrolysis, and ornithine decarboxylation.

Only the last three tests were used to biotype the Haemophilus isolates. All reactions were complete after four hours' incubation at $37^{\circ} \mathrm{C}$ in air. Reproducibility was checked with every test batch using $H$ influenzae NCTC biotypes $\mathrm{I}-\mathrm{V}$ as control organisms.

\section{Results}

The 200 Haemophilus species investigated comprised $193 \mathrm{H}$ influenzae, five $H$ parainfluenzae, and two $H$ parahaemolyticus. The sources of these isolates were sputum ( $n=159)$, blood or cerebrospinal fluid $(n=26)$, eye $(n=7)$, tracheal aspirate $(n=5)$, and antral washings $(n=3)$. Using " $x$ " and " $v$ " factor growth dependence as the standard method of identification, both kits performed well in the identification of isolates, and $100 \%$ sensitivity and specificity were achieved. There were few disagreements in biochemical reactions, none of which affected identification of the isolates. Three strains of $H$ influenzae atypically gave positive sucrose reactions, two with the RIM kit and one with the RAPID NH kit, while giving typical negative reactions with the other kits. One other strain gave an atypical negative dextrose reaction in the RIM kit, while giving typical positive glucose reactions in the Rapid NH and API kits. Also of note was the failure of the Rapid NH kit to show a positive reduction of resazurin to resorfin for any isolate.

The distribution of the $H$ influenzae biotypes as determined by the modified API method ${ }^{5}$ is shown in table 1. Comparison results of biotyping using the two rapid methods are shown in table 2. These data show $100 \%$ correlation with the indole reaction in both kits and with the ornithine reaction in the Rapid NH kit. The

Table 2 Correlation of biotyping reactions of 200 Haemophilus spp for RIM kit and Rapid NH kit compared with API $10 S$ kit

\begin{tabular}{|c|c|c|c|c|c|c|}
\hline & \multicolumn{2}{|c|}{ Indole } & \multicolumn{2}{|c|}{ Urea } & \multicolumn{2}{|c|}{ Ornithine } \\
\hline & + & - & + & - & + & - \\
\hline $\begin{array}{l}\text { API 10S } \\
\text { RIM } \\
\text { RAPID NH }\end{array}$ & $\begin{array}{l}149 \\
149 \\
(0) \\
149 \\
(0)\end{array}$ & $\begin{array}{l}51 \\
51 \\
(0) \\
51 \\
(0)\end{array}$ & $\begin{array}{l}176 \\
178 \\
(2) \\
137 \\
(1)\end{array}$ & $\begin{array}{l}24 \\
22 \\
(0) \\
63 \\
(39)\end{array}$ & $\begin{array}{l}94 \\
58 \\
(3) \\
94 \\
(0)\end{array}$ & $\begin{array}{l}106 \\
142 \\
(39) \\
106 \\
(0)\end{array}$ \\
\hline
\end{tabular}

$(0)=$ false results. poor performance of the Rapid NH urea reaction could be significantly improved if a light orange colour reaction was read as a positive result, but this is specifically not advised by the manufacturer. Reading the light orange reaction as positive, 160 were positive, with no false positive results, and 40 were negative with 16 false negative results. There were insufficient numbers of species other than $H$ influenzae to assess the biotyping ability of the two kits for other species, although the biochemical reactions of the seven non- $H$ influenzae isolates are included. Overall, 42 strains of $H$ influenzae were wrongly biotyped by the RIM kit and 40 by the Rapid NH kit. The control organisms were correctly identified and biotyped by all three methods.

\section{Discussion}

Rapid method (one to 4 hours) biochemical tests on bacteria depend on preformed substrate and constitutive rather than inducible enzymes for reliable reactions and are not growth dependent. Although several such kits have been reported to work well, ${ }^{89}$ others are reported to have problems with the indole or ornithine reactions. ${ }^{7}$ The poor performance of the RIM ornithine and Rapid NH urea reactions of these kits precludes their use as reliable rapid biotyping systems even after considerable collaboration with the manufacturer's agents and retesting of freshly delivered supplies. The problem therefore probably lies in the quality or quantity of substrate base, buffer, indicator; or other component quality rather than in the test enzyme, as these reactions worked well in the other kits. The light orange reaction in the Rapid NH urea reaction, for example, indicates a reaction process being detected but not sufficiently strong to give a wholly reliable result. Similarly, the RIM ornithine reaction wells showed a yellow to light green change with some expected positive strains, but this was not sufficiently strong to be scored as a dark green to blue positive reaction, as recommended by the manufacturer.

One isolate thought initially to be nontypable or biotype VIII (urea negative) by API and Rapid NH, but type III (urea positive) by the RIM method was confirmed as type III by the Public Health Reference Laboratory at Oxford, but under the constraints of the study protocol it was treated as urea negative or biotype VIII. The urea reaction was consistently negative on repeated testing by API and consistently positive in Oxford using BDH Analar urea.

The proportions within each biotype and associations of biotype with site of infection were found in the Northern Ireland isolates to be similar to those of previous United Kingdom studies, ${ }^{6}$ although other studies have shown biotype distribution to vary with geographic location. ${ }^{1011}$

The cost of the Rapid NH is four times that of the API 10S on the basis of a per isolate test, although the more extensive number of reactions of the Rapid NH permits the speciation of 
several more genera than the RIM kits. The RIM $\mathrm{H} 1$ and $\mathrm{H} 2$ kits cost twice that of the API $10 S$, so although they give more rapid results for $H$ influenzae, they offer no advantage in terms of cost or reliability.

The kits performed well for identification purposes and could be very useful in giving preliminary identification of important isolates on a one to four hour basis-for example, in cases of meningitis, septicaemia, or epiglottitis-while waiting for more routine overnight growth dependent identification, although a heavy inoculum (McFarland No 3) is required. For the biotyping application, however, obtaining results earlier than overnight is of doubtful advantage in clinical practice. Therefore, on the grounds of cost, reliability, and usefulness we would recommend the use of the API 10S system for the biotyping of strains of $H$ influenzae in preference to the Rapid $\mathrm{NH}$ and RIM Haemophilus kits.

We acknowledge the help of Mercia Diagnostics and Cortecs Diagnostics Ltd, for the supply of the Rapid NH and RIM kits, respectively.
1 Kilian $M$, Sorensen I, Frederickson W. Biochemical characteristics of 130 recent isolates from Haemophilus influenzae meningitis. J Clin Microbiol 1978;9:409-12.

2 Oberhofer TR, Back AE. Biotypes of Haemophilus encountered in clinical laboratories. J Clin Microbiol 1979;10:168-74. 3 Kilian M. Haemophilus. In: Lennette EM, ed. Manual of
clinical microbiology. 4th Edition. Washington DC: American Society for Microbiology, 1985:387-93.

4 Sottnek F, Albritton WL. Haemophilus influenzae biotype VIII. J Clin Microbiol 1984;20:815-6.

5 Mehtar S, Aminiafshar S. Biotyping of Haemophilus species with API 10S. API update 1985:8.

6 Leinonen M, Sivonen A. Serological grouping of Meningococci and encapsulated Haemophilus influenzae strains by latex agglutination. J Clin Microbiol 1979;10:404-8.

7 Mehtar S, Aminiafshar S. Biotyping Haemophilus using API 10S-an epidemiological tool? J Clin Pathol 1983;36:96-9.

8 Edberg SC, Melton E, Singer JM. Rapid Biochemical Characterisation of Haemophilus species by using the Micro-ID. J Clin Microbiol 1980;11:22-6.

9 Back AE, Oberhafer TR. Use of the Minitek system for Biotyping Haemophilus species.J Clin Microbiol 1978;7:312-3.

10 Kamme C. Haemophilus influenzae: Correlation between biotypes and B-lactamase production. Acta Pathol Microbiol Scand (B) 1980;88:261-4.

11 Tsai WC, Wu JJ. Serotypes and biotypes and antibiotic susceptibility of Haemophilus influenzae encountered in clinical laboratory in Taiwan. J Clin Microbio 1979;12:140-8. 